\title{
OS SINTOMAS DEPRESSIVOS E AS RELAÇÕES SOCIAIS NA TERCEIRA IDADE ${ }^{\star}$
}

Marilia Ramos ${ }^{\star}$

\begin{abstract}
RESUMO
O artigo testa a hipótese, originada da teoria da Equidade e da teoria da Integração Social, sobre o efeito das trocas sociais nos sintomas depressivos dos idosos. A base de dados amostral original é formada por 871 idosos pesquisados no Rio Grande do Sul em 1995, dentre os quais, 551 foram re-entervistados em 1999. Análises multivariadas transversais e longitudinais estimaram efeito de duas dimensões das relações sociais (as trocas com familiares e a integração social) nos sintomas depressivos. Os resultados indicam que o desbalanço nas trocas aumenta os sintomas depressivos, e a integração social contribui para um decréscimo dos mesmos.
\end{abstract}

Palavras-chave: Depressão. Idosos. Relações sociais. Trocas sociais. Integração social.

\section{DEPRESSIVE SYMPTONS AND SOCIAL RELATIONSHIPS AMONG ELDERLY POPULATION}

\begin{abstract}
This article tests the hypotheses from equity theory and social integration theory regarding the effect of social relationships on depressive symptoms. The expected result is that social relationships tend to decrease the number of depressive symptoms. The study was based in two points (waves) in time. The baseline sample consists of 871 subjects interviewed in 1995, among those, 551were reinterviewed in 1999. Cross-sectional and longitudinal multivariate regression models estimate the effect of two dimensions of social relationships (i.e., social exchanges and social integration) on depressive symptoms. The results indicate that unbalanced exchange increases depressive symptoms, and social integration decreases depressive symptoms.

* Essa pesquisa foi financiada pela Purdue Research Foundation (PRF) EUA, pela CAPES, pela Universidade de Santa Cruz do Sul, (UNISC) e pela Fapergs (Fundação de Amparo a Pesquisa do Estado do Rio Grande do Sul). Agradecemos aos professores/pesquisadores, Dr. Janet Wilmoth, Dr.Ken Ferraro, Dr. Harry Potter, e Dra. Debra Street pelos comentários nas versões iniciais dessa pesquisa.

$\star \star \mathrm{PhD}$ em Sociologia pela Purdue University EUA, professora/pesquisadora da Universidade de Santa Cruz do Sul, Brasil. Endereço: Av. Independência 2293/104, Santa Cruz do Sul, Rio Grande do Sul, Brasil, CEP 96815-900.

E-mail: ramos@unisc.br
\end{abstract}


Keywords: Depressive symptoms. Old ageds. Social relations. Social exchanges. Social integration.

\section{INTRODUÇÃO}

A população idosa representa cerca de $9 \%$ da população Brasileira e é estimado que esse segmento da população representará 24\% no ano 2050 (IBGE, 2000). Para 2004 o IBGE estimou o total de idosos no Brasil em torno de 14,7 milhões de pessoas. Isso gera preocupações por vários razões:

Primeiro o padrão de vida entre os Brasileiros de todas as idades permanece cada vez mais baixo, sendo que $2 / 3$ da população vive abaixo da linha de pobreza e em péssimas condições de saúde (KALACHE; VERAS; RAMOS, 1987). Segundo, a família é a fonte primeira de cuidados, mas as relações familiares estão mudando cada vez mais em função da modernização. Como resultado, os idosos na maioria das vezes não recebem o cuidado que esperam receber se seus familiares. Terceiro, existe uma visível falta de oportunidades, para os idosos no Brasil, de poder participar em atividades sociais fora da esfera familiar (DEBERT, 1999), fato esse que limita a participação deles em um leque mais amplo de relações sociais.

O principal objetivo dessa pesquisa é identificar o efeito das relações sociais nos sintomas depressivos entre idosos no Sul do Brasil. As evidências sugerem que a depressão está muito presente entre a população idosa no Brasil. Entre pessoas com 60 anos ou mais no Brasil 48\% sofrem de alguma forma de depressão (RAMOS; SAAD, 1990). Entretanto, relativamente muito pouco é conhecido sobre os fatores sociais que influenciam na depressão nessa população (JANG. et al., 2000).

\section{A teoria da INTEGraÇão SOCIAL E A teOria das trocas}

Esse estudo utiliza duas correntes teóricas para entender a relação entre relações sociais e sintomas depressivos entre os idosos no contexto Brasileiro. Primeiro, se baseia na Teoria da Integração Social. Como apontado por Pillemer \& Glasgow (2000), a integração social não está consistentemente definida na literatura. Ela é freqüentemente usada para se referir aos laços interpessoais, tais como número de membros numa rede de relações de uma pessoa ou número de papéis sociais que um indivíduo possui. Entretanto, este conceito também é referido ao pertencimento ou envolvimento social, o qual é indicado pelo volume dos laços sociais tais como casamento e contatos com amigos e familiares (BARRERA, 1986). Essas formas de integração social são importantes e devem ser consideradas nessa pesquisa por que: (a) a falta de integração social rouba o sentido da vida, gerando depressão e stress; (b) a integração social fortalece o acesso as fontes de ajuda e (c) a integração social fornece um contexto dentro do qual relações de suporte e ajuda podem se desenvolver fora da família (GEORGE, 1996). 
Por isso, se espera que quanto maior a integração social menor o número de sintomas depressivos. Essa hipótese tem sido corroborada por várias pesquisas, por exemplo, pesquisas nos EUA e em outros países desenvolvidos sugerem que as relações sociais estão associadas com o bem estar psicológico na fase adulta e do envelhecimento (ANTONUCCI, 1990; HOUSE, 1981; GEORGE, 1996).

Esse estudo também se baseia na Teoria da Equidade, a qual é derivada da Teoria das Trocas Sociais (BLAU, 1964; DOWD, 1975). Essa perspectiva teórica enfoca a equidade das trocas entre atores sociais. Pesquisas com idosos nos Estados Unidos indicam que receber ajuda está positivamente relacionado com os sintomas depressivos, enquanto que fornecer ajuda está negativamente relacionado (LIANG; KRAUSE; BENNETT, 2001). Entretanto, não está claro como o balanço das trocas influencia os sintomas depressivos porque a relação pode tomar uma das três diferentes formas apresentadas a seguir:

Primeiro, poderia haver uma relação negativa entre as trocas não balanceadas e sintomas depressivos, Krause (1995) sugere que ser sobrebeneficiado (isto é, receber mais do que se fornece) pode ser positivo em termos utilitários. Idosos podem sentir que os jovens têm de retribuir por ajudas prévias que seus pais lhes deram na infância, por exemplo. Segundo, poderia haver uma relação positiva entre trocas não balanceadas e sintomas depressivos. Alguns têm notado que: ser sobrebeneficiado pode contribuir para a percepção de certa perda de autonomia e sentimentos de dependência, os quais podem levar a insatisfação, baixa auto-estima e aumento dos sintomas depressivos (CICIRELLI, 1990; STOLLER, 1985). Nessa circunstância, em comparação com aqueles que têm trocas balanceadas, ser sobre-beneficiado aumentaria os sintomas depressivos, enquanto que ser sub-beneficiado decresceria os sintomas depressivos. A partir da perspectiva da Teoria da Equidade, o aspecto mais importante de uma relação social seria o balanço das trocas entre os atores. Qualquer tipo de desbalanço nas trocas seria problemático. Então, a Teoria da Equidade prevê maior número de sintomas depressivos entre idosos que são sobre ou sub-beneficiados, em comparação com aqueles que experimentam trocas mais balanceadas.

Apesar de a literatura conter numerosos estudos que examinam a reciprocidade e várias medidas de bem estar mental (DAVEY; EGGEBEEN, 1998; INGERSOLL-DAYTON; ANTONUCCI, 1988; MCCULLOCH, 1990; STOLLER, 1985), estamos cientes apenas de dois estudos que explicitamente testam a relação entre o balanço nas trocas e os sintomas depressivos (LEE; NETZER, 1995; LIANG; KRAUSE; BENNETT, 2001). Os resultados são consistentes com segunda explicação, sobre-beneficio está relacionado com maior número de sintomas depressivos, enquanto sub-beneficio com menor número de sintomas depressivos. Entretanto, pelo fato dos dois estudos serem baseados em amostras nos Estados Unidos, é importante testar esta relação com dados de diferentes contextos culturais.

Com base nas duas perspectivas teóricas e pela evidência fornecida por outras pesquisas se espera que as relações sociais tenham influência significativa nos sintomas depressivos dos idosos no Sul do Brasil. Em particular, tem-se 
como hipótese que: controlando-se por características demográficas, status socioeconômico e características de saúde, a) trocas não balanceadas aumentam os sintomas depressivos e b) que a integração social reduz os sintomas depressivos.

\section{A metodologia da Pesquisa e as VARIÁveis PeSQuisadas}

O estudo envolveu uma amostra representativa de idosos da região CentroOriental do Rio Grande do Sul. De acordo com o Índice para o Desenvolvimento Humano criado pelas Nações Unidas em 1996, o Rio Grande do Sul é um dos Estados mais desenvolvidos no Brasil. A expectativa de vida nesse Estado é maior, em 3,26 anos, que a média nacional (UNITED NATIONS, 1998). A amostra desse estudo foi composta por 10 municípios aleatoriamente selecionados de um total de 36 municípios da região Centro-Oriental do Rio Grande do Sul, baseada em estratos de acordo com o tamanho da população e a atividade econômica. Quinze por cento dos setores censitários foram selecionados em cada município para comporem a amostra. Um mínimo de oito setores censitários foi usado para cada município para capturarmos a variabilidade de cada município e então oito domicílios foram selecionados para cada setor. Uma pessoa com 60 anos ou mais foi entrevistada por domicílio. Os dados foram coletados através de um questionário fechado com 121 questões fechadas. Este artigo apresenta os resultados de duas pesquisas feitas uma em 1995 e outra em $1999^{1}$ com os mesmos pesquisados, caracterizando-se então como um estudo longitudinal. Os respondentes do ano base (1995) foram re-entrevistados em 1999. Entre os 871 da amostra original (1995), 551 (63,4\%) responderam em 1999. Entre aqueles que não responderam, 118 morreram, 51 se recusaram, 42 estavam em asilos, hospitais ou incapazes de responder e 109 não foram localizados. A tabela 1 apresenta as características descritivas da amostra. Os dados são consistentes com os dados da população dessa região, sugerindo que a amostra é representativa.

\section{VARIÁVEL DEPENDENTE: SINTOMAS DEPRESSIVOS}

Doze itens, com categorias de resposta dicotômicas ( $0=$ não e $1=\operatorname{sim})$ são usadas para medir os sintomas depressivos em 1995 e 1999. Estes itens foram desenvolvidos e testados numa pesquisa desenvolvida pelo Conselho Estadual do Idoso do RS (1997). A variável dependente é uma escala aditiva que vai de 0 até 12 , com números mais altos indicando maior número de sintomas depressivos. A média do número de sintomas depressivos é de 2,64 em 1995 e de 2,72 em 1999. Destacamos que o coeficiente alpha de fidedignidade das escalas é igual a $0,85 \mathrm{em}$ 1995 e 0,80 em 1999 e por essa razão nossa escala parece ser uma medida adequada para mensurar os sintomas depressivos no contexto cultural do Sul do Brasil.

\section{VARIÁVEIS INDEPENDENTES: RELAÇÕES SOCIAIS}

Esse estudo mensura duas dimensões das relações sociais: as trocas sociais e a integração social. As trocas sociais são mensuradas pela freqüência da ajuda recebida ou fornecida de familiares em três áreas: emocional, financeira e instru- 


\section{Tabela 1}

Características descritivas da amostra, 1995 e 1999

\begin{tabular}{|c|c|c|}
\hline Variáveis & $\begin{array}{l}1995 \\
(\mathrm{~N}=871)\end{array}$ & $\begin{array}{l}1999 \\
(\mathrm{~N}=551)\end{array}$ \\
\hline Idade (amplitude $=60-95)^{*}$ & $\begin{array}{l}70,08 \\
(7,03)\end{array}$ & $\begin{array}{l}73,06 \\
(6,38)\end{array}$ \\
\hline Mulheres (=1) &, 71 & ,73 \\
\hline Não broncos &, 10 &, 08 \\
\hline \multicolumn{3}{|l|}{ Arranjos de vida } \\
\hline Vive somente c/ cônjuge & ,26 & ,23 \\
\hline Vive sozinho** &, 18 & ,20 \\
\hline Vive $\mathrm{c} /$ cônjuge e filhos &, 15 &, 12 \\
\hline Vive com filhos &, 12 &, 14 \\
\hline Vive com outras pessoas** & ,29 &, 31 \\
\hline \multicolumn{3}{|l|}{ Estado Civil } \\
\hline Casado (inclui “juntados) * & ,44 & ,41 \\
\hline Viúvo** &, 47 & ,49 \\
\hline Divorciado (inclui separados) &, 05 &, 06 \\
\hline Nunca casou &, 04 &, 04 \\
\hline \multicolumn{3}{|l|}{ Educação formal } \\
\hline Fundamental incompleto ou sem instrução & ,64 & ,63 \\
\hline Ensino fundamental completo &, 25 & ,26 \\
\hline Médio incompleto & ,06 &, 06 \\
\hline Médio completo (ou superior) &, 05 &, 05 \\
\hline \multicolumn{3}{|l|}{$\underline{\text { Ocupação }}$} \\
\hline Ocupação não remunerada &, 21 &, 21 \\
\hline Ocupação de baixo status & ,49 &, 53 \\
\hline Ocupação de status médio &, 04 &, 03 \\
\hline Ocupação de alto status &, 25 & ,23 \\
\hline $\begin{array}{l}\text { Renda per capita (amplitude }=\mathrm{R} \$ 15,5- \\
\mathrm{R} \$ 2500)\end{array}$ & $\begin{array}{l}\mathrm{R} \$ 196,07 \\
(23,50)\end{array}$ & $\begin{array}{l}194,51 \\
(23,89)\end{array}$ \\
\hline \multicolumn{3}{|l|}{ Estado de saúde } \\
\hline \# Comportamentos de risco (intervalo=0-6) & $\begin{array}{l}, 32 \\
(, 11)\end{array}$ & $\begin{array}{l}, 30 \\
(, 12)\end{array}$ \\
\hline \# Condições crônicas (intervalo $=0-8$ ) $* *$ & 2,07 & 3,11 \\
\hline
\end{tabular}

Nota: desvio padrão entre parênteses,

* Diferença significante entre 1995 e 1999 proporções ou médias, $p<, 05$,

** Diferença significante entre 1995 e 1999 proporções ou médias, $p<, 01$

Revista do Departamento de Psicologia - UFF, v. 19 - n. 2, p. 397-410, Jul./Dez. 2007 
mental. O balanço das trocas, o qual mensura o conceito de reciprocidade, está baseado em duas variáveis: ajuda recebida e ajuda fornecida. A construção da cada tipo de ajuda é explicada para facilitar a compreensão das medidas referentes à ajuda recebida e ajuda fornecida. A ajuda emocional envolve a questão sobre a freqüência de ajuda emocional recebida (ou fornecida), tais como conselhos. As opções de respostas variam de $0=$ nunca até $4=$ diariamente. A ajuda financeira está baseada numa questão sobre a freqüência da ajuda financeira recebida ou fornecida, com as mesmas opções de resposta ( $0=$ nunca e $4=$ diariamente).

Diferente dos demais tipos, a ajuda instrumental é um índice aditivo, o qual pergunta sobre a freqüência com que os sujeitos recebem ou fornecem aos familiares os seguintes itens: (a) roupas, (b) moradia, (c) comida e (d) remédios ou cuidados pessoais. A freqüência com a qual os sujeitos recebem ou fornecem os quatro tipos de ajuda instrumental é codificada como $0=$ nunca até $4=$ diariamente. Então, o escore máximo para o total de ajuda instrumental é 16 (isto é, quatro itens vezes quatro níveis de freqüência). A medida da ajuda instrumental fornecida contém um item adicional: ajudar como babá. Isso torna o escore máximo para ajuda instrumental fornecida igual a 20 (cinco itens vezes quarto níveis de freqüência). Ambas escalas de ajuda instrumental (recebida e fornecida) são divididas pelo número de itens da escala para restringir o intervalo de 0 até 4 , o qual se torna comparável com os intervalos dos demais tipos de ajuda. Para todas as escalas de troca, números altos indicam que mais ajuda está sendo recebida ou fornecida. O total de ajuda fornecida é a soma de do total de ajuda emocional ( 0 até 4$)$, total de ajuda financeira ( 0 até 4$)$ e total de ajuda instrumental (0 até 4$)$. Então o total de ajuda fornecida varia de 0 até 12 ), com 0 indicando nenhuma ajuda fornecida em qualquer um dos três tipos de ajuda. $\mathrm{O}$ total de ajuda recebida é medido da mesma forma que o total de ajuda fornecida.

Semelhante a outros autores (ROOK, 1987; WILMOTH, 2000), medimos o balanço das trocas subtraindo o total de ajuda fornecida do total de ajuda recebida. Apesar dessa escala teoricamente estar entre -4 até +4 , o intervalado observado na amostra está entre -3 e +3 . Onde escores negativos indicam que os sujeitos são sub-beneficiados, escores positivos indicam que são sobre-beneficiados e escores iguais a zero ou perto de zero indicam trocas balanceadas ou ausência de trocas.

A intensidade nas trocas, a qual é uma medida da integração social, consiste na soma de todos os itens trocados (total recebido mais total fornecido) e vai de 0 até 24 . Se espera que essa variável tenha um efeito negativo nos sintomas depressivos porque pessoas com altos valores estão mais integradas e por isso devem estar menos depressivas.

A integração social na velhice é manifestada através de uma variedade de indicadores (PILLEMER; GLASGOW, 2000). Esse estudo inclui quarto medidas adicionais da integração social: contato com amigos $(1=\operatorname{sim})$, contato com familiares $(1=\operatorname{sim})$, arranjos de vida (vive somente com cônjuge, sozinho, com cônjuge e filhos, somente com filhos e com outras pessoas), e o estado civil ( 1 = casado). 
Finalmente, a análise inclui uma medida referente à satisfação com as relações familiares $(1$ = satisfeito). Essa medida controla pela avaliação subjetiva dos pesquisados com relação as relações sociais que eles travam com os familiares.

\section{VARIÁVEIS de CONTROLE (MEDIDAS EM 1995)}

Várias variáveis medidas em 1995 são incluídas como controles por causa de sua relação com sintomas depressivos. A idade é medida pelo número de anos desde o nascimento, o gênero é uma variável dicotômica ( $1=$ mulheres), raça é dicotomizada $(0=$ brancos $)$, o status socioeconômico é medido através de três indicadores: a educação contém quatro categorias, fundamental incompleto, fundamental completo (referência), médio incompleto e médio completo. A ocupação contém quatro categorias: ocupação não assalariada (categoria de referência a qual inclui aqueles que nunca trabalharam ou estão desempregados), ocupação de baixo status (ex: trabalhadores braçais, trabalhadores domésticos e pequenos agricultores), ocupação de médio status (ex: secretárias e trabalhadores do setor público), e ocupação de alto status (ex: profissionais com curso superior ou donos de grandes negócios). ${ }^{2}$

A renda per capita é medida pelos Reais ganhos por mês na residência dividido pelo total de pessoas que vivem naquela residência.

Uma outra variável de controle importante diz respeito aos comportamentos de risco, os quais apresentam conseqüências negativas para a saúde mental (IDLER; ANGEL, 1990). Os comportamentos de risco são medidos por 6 variáveis dicotômicas, as quais medem se o sujeito é fumante ou desenvolve comportamentos de risco associados ao abuso no consumo do álcool. Estes itens são usados para criar uma escala aditiva que vai de 0 até 6 , a qual mede o número total de comportamentos de risco.

Os indicadores de morbidade foram coletados no ano base (1995) através das seguintes questões: "Você teve (problema específico) em tratamento nos últimos 6 meses?" ( $0=$ não e $1=\operatorname{sim})$. Os 13 indicadores de morbidade são divididos em 2 indicadores aditivos para especificar os efeitos de tipos gerais de problemas de saúde nos sintomas depressivos (FERRARO; WILMOTH, 2000). A escala para medir as condições crônicas de saúde inclui reumatismo, bronquite, problemas de rins, varizes, problemas de coluna, úlceras ou gastrites, problemas urinários e problemas de pele. A escala para as condições sérias de saúde inclui aspectos mais ameaçadores da saúde: pressão alta, câncer, diabete, AVCs e problemas de coração.

As limitações físicas são medidas por um conjunto de sete questões. As seis primeiras se referem à necessidade de ajuda para realizar as seguintes atividades: cozinhar, limpar a casa, tomar remédio, fazer a higiene pessoal, comer e se mover $(0=$ não e $1=\operatorname{sim})$. A sétima questão pergunta se o respondente caminha sem dificuldade, mesmo subindo escadas $(0=$ sem problemas e $1=$ com algum problema). Os itens individuais foram somados para criar uma escala que vai de 0 até 7 , com números altos indicando maior número de limitações físicas. 


\section{CONDICIONANTES DOS SINTOMAS DEPRESSIVOS}

Consistente com as hipóteses a tabela 2 mostra que existe uma associação significativa entre os sintomas depressivos e as relações sociais. Apesar das variáveis ajuda recebida e ajuda fornecida não serem significativas para os sintomas depressivos; o balanço das trocas está positivamente associado com os sintomas depressivos em ambos os períodos (1995 /1999).

A tabela 2 indica também que aqueles que estão socialmente mais integrados relataram menor número de sintomas depressivos. Especificamente, a intensidade nas trocas está associada com número pequeno de sintomas depressivos, enquanto que viver com filhos somente ou não ser casado está relacionado com maior número de sintomas depressivos. Em complemento a satisfação com as relações familiares está associada com menor número de sintomas depressivos.

Tabela 2 Associações entre sintomas depressivos e as variáveis independentes

Sintomas Depressivos

Características das relações sociais

$\underline{1995}$

1999

Trocas Sociais

Ajuda recebida ${ }^{2}$

, $021 \quad-, 065$

Ajuda fornecida ${ }^{a}$

, 053

$-, 089$

Balanço das trocas (ajuda recebida - ajuda

fornecida) ${ }^{\mathrm{a}}$

,738* , 701*

Integração Social

Intensidade das Trocas ${ }^{\mathrm{a}}$

$-, 482 * \quad-, 423 *$

Contato com amigos ${ }^{\mathrm{a}}$

$-, 014 \quad-, 032$

Contato com familiares ${ }^{\mathrm{a}}$

, 033

, 034

Arranjos de Vida ${ }^{\mathrm{b}}$

Vive somente com cônjuge (referência)

$2,35 \quad 2,46$

Vive sozinho

$2,62,81 *$

Vive com filhos

$2,68^{*} \quad 2,01^{*}$

Vive com cônjuge e filhos

$2,78 \quad 2,82$

Vive com outras pessoas

$2,84 \quad 2,87$

Estado Civil $^{\mathrm{c}}$

Casado (referência)

$2,61 * \quad 2,58^{*}$

Não casado

2,86

2,98

Avaliação subjetiva

Satisfação com as relações familiares ${ }^{\mathrm{a}}$

$-, 640 * *$

$-, 645^{* *}$

${ }^{\mathrm{a}}$ Coeficiente de correlação de Pearson ${ }^{\mathrm{b}}$ Média dos sintomas depressivos por arranjos de vida, desvio padrão entre parenteses, significância baseada em Análise de Variância com teste de tukey t-tests indicando diferenças entre o grupo de referência e as outras categorias dos arranjos de vida. ${ }^{c}$ Média dos sintomas depressivos por estado civil, desvio padrão entre parenteses, significância baseada no teste $t$ para diferença de médias, ${ }^{*} \mathrm{p}<, 05 ; * * \mathrm{p}<, 01 ; * * * \mathrm{p}<, 001$ 


\section{O EFEITO DAS RELAÇÕES SOCIAIS NOS SINTOMAS DEPRESSIVOS EM UM MOMENTO NO TEMPO-ANÁLISE TRANSVERSAL}

A análise de regressão transversal nos mostrou que, apesar dos efeitos da ajuda recebida e da ajuda fornecida não serem significativos, a interação entre as duas dimensões está relacionada de forma significativa com os sintomas depressivos. Isso sugere que o efeito da ajuda recebida nos sintomas depressivos varia de acordo com níveis de ajuda fornecida. Em outras palavras, os sintomas depressivos são menores quando os idosos que recebem ajuda podem retribuí-las. A intensidade das trocas tem um efeito negativo no número de sintomas depressivos. Isto é, idosos que estão envolvidos em trocas mais freqüentes com familiares apresentam menor número de sintomas depressivos que os demais.

É interessante notar que os arranjos de vida e o estado civil afetam os sintomas depressivos. Estar casado contribui para decréscimos nos sintomas depressivos, enquanto não morar com cônjuge aumenta substancialmente o número de sintomas depressivos. Em complemento, a satisfação com as relações familiares faz com que com os depressivos decrescerem. As mulheres idosas tendem a ser mais depressivas que os homens idosos e as condições crônicas de saúde, os comportamentos de risco e as limitações físicas aumentam o número de sintomas depressivos.

\section{O EFEITO DAS RELAÇões SOCIAIS ATRAVÉS DO TEMPO -ANÁLISE LONGITUDINAL}

Os modelos longitudinais estimam os sintomas depressivos em 1999 como uma função dos mesmos medidos em 1995, das relações sociais e das variáveis de controle medidas em 1995.

As análises longitudinais indicam que os sintomas depressivos no ano base têm grande impacto nas mudanças dos sintomas depressivos através do tempo (quatro anos depois). Isto quer dizer que respondentes com grande número de sintomas depressivos no ano base (1995) são mais propensos a ter um acréscimo nos mesmos através do tempo.

Os efeitos das trocas sociais nesses modelos longitudinais são semelhantes aqueles dos modelos transversais. A interação entre ajuda recebida e ajuda fornecida é significativa e negativa, indicando que a mudança nos sintomas depressivos é menor quando aqueles que recebem ajuda são capazes de retribuir. A relação entre o balanço nas trocas e os sintomas depressivos indica que aqueles respondentes que recebem mais do que fornecem e vice versa apresentam mais sintomas depressivos com o passar do tempo que aqueles que trocam de forma balanceada. Mudanças nos sintomas depressivos são menores entre idosos que estão mais envolvidos nas trocas.

A integração social tende a suprimir o número de sintomas depressivos. Idosos que são casados apresentam pequenas mudanças nos sintomas depressivos que aqueles que não são casados, enquanto que aqueles que vivem sozinhos apre- 
sentam mais acréscimos nos sintomas depressivos que aqueles que vivem com cônjuge. Em complemento, a satisfação com as relações familiares contribui para o decréscimo dos sintomas depressivos através do tempo. Semelhante aos resultados da análise transversal, mulheres e aqueles em condições precárias de saúde têm acréscimos substanciais nos seus sintomas depressivos através do tempo.

\section{Conclusões}

Essa pesquisa oferece evidência adicional de que as relações sociais em geral, e o balanço das trocas e a integração social em particular, têm implicações para a saúde mental na velhice. Entretanto, em contraste com algumas pesquisas prévias que encontraram uma relação positiva entre trocas não balanceadas e sintomas depressivos (LEE; NETZER, 1995; LIANG; KRAUSE; BENNETT, 2001), essa pesquisa encontrou uma situação na qual qualquer tipo de desbalanço nas trocas está relacionado com grande número de sintomas depressivos. Então, idosos Brasileiros que recebem mais do que fornecem, ou vice versa, têm mais sintomas depressivos em um ponto no tempo e maior número nos sintomas depressivos através do tempo que aqueles idosos que travam trocas balanceadas com familiares.

Essas evidências são consistentes com as previsões da Teoria da Equidade e reforçam a idéia de que a saúde mental é negativamente afetada pela falta de interdependência entre membros de uma família (STOLLER, 1985; RAMOS, 1992). E indicam também que o efeito da ajuda fornecida nos sintomas depressivos varia de acordo com níveis de ajuda recebida. Os resultados mostram que a intensidade nas trocas está negativamente relacionada com os sintomas depressivos. Esses resultados juntos enfatizam o potencial das trocas balanceadas e da sua intensidade para reduzir os sintomas depressivos entre a população idosa no Sul do Brasil.

Um resultado não esperado foi que o contato com amigos e com familiares não teve um efeito significativo nos sintomas depressivos transversalmente e nem longitudinalmente. Isso sugere que a simples presença de um contato social, sem nenhum tipo de troca, não reduz os sintomas depressivos. Isso pode se dar em função das expectativas culturais com relação às trocas. Trocas com a família são esperadas por causa do caráter de obrigatoriedade das relações familiares (GEORGE, 1996; NERI, 1993; ROOK, 1987).

Reforçando a teoria da integração social, os modelos de regressão indicam que a intensidade nas trocas (a qual é uma medida da integração social), os arranjos de vida e o estado civil foram consistentemente relacionados com os sintomas depressivos em um ponto no tempo (análise transversal) e nas mudanças dos sintomas depressivos (análise longitudinal). Em função disso concluímos que o balanço nas trocas e a integração social são importantes para a saúde mental dos idosos. Em complemento, a avaliação subjetiva dos idosos Brasileiros com relação às relações familiares se apresentou relacionada com os sintomas depressivos. Isso demonstra a importância de levarmos em conta aspectos objetivos e subjetivos das relações sociais quando estudamos a saúde mental e o envelhecimento. 
Quanto às limitações deste estudo, apesar dele incluir várias medidas das trocas sociais, salientamos que ele não é capaz de dar conta de toda a complexidade das trocas sociais. O questionário base (1995) não especifica com quais familiares os idosos interagem ou trocam (exemplo: se com filhos, primos etc...). Ele também não contém informação detalhada sobre com quais filhos os idosos trocam alguma coisa (exemplo: filhos ou filhas, filhos geograficamente próximos ou não) e nem o grau de concentração das redes sociais. Essa pesquisa não incluiu trocas sociais com amigos por causa das limitações dos dados, as quais foram causadas pela relativa raridade com que os pesquisados se relacionam com amigos. Conseqüentemente, essa pesquisa não pode fornecer insights quanto aos diferentes efeitos das trocas com filhos, com outros parentes, com amigos ou vizinhos separadamente.

Finalmente, é importante enfatizar a contribuição deste estudo para as políticas públicas relacionadas aos idosos. Existe uma ênfase implícita na fragilidade dos idosos em certas políticas direcionadas a eles. Entretanto, os resultados confirmam que o bem estar na velhice não está simplesmente relacionado com o estado de saúde. As relações sociais, em geral e o balanço das trocas em particular, também têm um importante efeito no bem estar dos idosos. Apesar desse fato, as políticas não têm levado em conta a importância da facilidade em poder trocar de forma balanceada. Essa pesquisa sugere que trocas balanceadas potencialmente previnem os idosos quanto a sentimentos negativos, tais como se sentirem um peso para a família ou se sentirem explorados por ela. Esse tipo de padrão de trocas está mais relacionado com resultados na saúde em contextos culturais onde valores utilitaristas prevalecem, tais como os países desenvolvidos do Ocidente e regiões tais como aquela tomada em consideração nesse estudo. Resultados positivos para saúde são facilitados pela integração social. Redes com laços fortes, por meio de trocas intensas, arranjos de vida e o casamento são essenciais para o bem estar mental dos idosos. Esse resultado é particularmente importante dado que idosos no Brasil não tendem a participar em atividades sociais fora da esfera familiar, por causa das limitadas opções para atividades sociais na comunidade em geral. Políticas públicas que encorajassem o envolvimento social e a formação de redes amplas de suporte social para os idosos poderiam ter um impacto positivo na saúde mental desse grupo, particularmente para aqueles que estão isolados por não terem parentes próximos, por terem baixo status socioeconômico ou por terem saúde precária.

Em geral, os resultados sugerem que programas para idosos foquem mais na promoção de atividades sociais ao invés de atividades meramente contemplativas. As políticas deveriam promover o senso de utilidade entre os idosos. Por exemplo através do trabalho voluntário em creches ou hospitais e mesmo em asilos. Essas políticas podem fazer os idosos se sentirem úteis num contexto cultural no qual valores utilitários são predominantes. Elas podem também ajudar outras pessoas necessitadas, tais como crianças abandonadas ou outros idosos com poucas redes de suporte social. Como conseqüência tais políticas podem promover o bem estar da população idosa enquanto contribuem para auxiliar outros segmentos da população. 


\section{Notas}

${ }^{1}$ Cabe destacar que dados os custos das pesquisas, em países do Terceiro Mundo trabalhar com informações de 5 ou mais anos atrás é algo aceitável, haja vista os Censos do IBGE que acontecem de 10 em 10 anos no Brasil. Tal fato não invalida o presente trabalho posto que o mesmo visa a apresentar tendências e não absolutizar resultados.

${ }^{2}$ Tal classificação foi feita com base nas classificações do IBGE

\section{REFERÊNCIAS}

ANTONUCCI, T, C. Social supports and social relationships. In: BINSTOCK, R. H.; GEORGE, L. K. (Ed.). Handbook of aging and the social sciences. New York: Academic Press, 1990. p. 205-226.

BARRERA, M. Distinctions between social support concepts, measures, and models. American Journal of Community Psychology, EUA, v. 14, n. 4, p. 413445, Aug. 1986.

BLAU, P. Exchange and power in social life. New York: Wiley, 1964.

CICIRELLI, V. G. Family support in relation to health problems of the elderly. In: BRUBAKER T. H. (Ed.). Family relationships in later life. $2^{\text {nd }}$ ed. Newbury Park, CA: Sage. 1990. p. 212-228.

DAVEY, A.; EGGEBEEN, D. J. Patterns of intergenerational exchange and mental health. Journal of Gerontology: Psychological Sciences, EUA, n. 53B, p. 86-95, 1998.

DEBERT, G. A Reinvenção da Velhice. São Paulo: Edusp, 1999.

DOWD, J, Aging as exchange: a preface to theory. Journal of Gerontology, EUA, v. 30, n. 5, p. 584-594, Sept. 1975.

FERRARO, K.; WILMOTH, J. M. Measuring morbidity in health surveys: disease counts, binary variables, and statistical power. Journal of Gerontology: Social Sciences, EUA, 2000, nº 55, p. 173-189.

GEORGE, L. Social factors and illness, In: BINSTOCK, R.; GEORGE, L. (Ed.). Handbook of aging and social sciences. New York: Academic Press, 1996. p. 229-252.

HOUSE, J. Work, stress and social support, Reading. PA: Addison-Wesley, 1981.

IBGE. Fundação Instituto Brasileiro de Geografia e Estatística. Censo Demográfico. 2000. 
IDLER, E.; ANGEL, R. Self-rated health and mortality in the NHANES-I Epidemiologic Follow-up Study. American Journal of Public Health, v. 80, n. 4, p. 446-452, 1990.

INGERSOLL-DAYTON, B.; ANTONUCCI, T, C. Reciprocal and nonreciprocal social support: contrasting sides of intimate relationships, Journal of Gerontology, EUA, n. 43, p. 65-73, 1988.

JANG, Y. et al. Psychosocial resources and predictors of depression among older adults in Korea: the role of sense of mastery, social network, and social support. Hallym International Journal of Aging, v. 2, p. 26-35, 2000.

KALACHE, A.; VERAS, R. P.; RAMOS, L. R. Aging of the world's population: a new challenge. Revista Saúde Pública, São Paulo, v. 21, n. 3, p. 200-210, jun. 1987.

KRAUSE, N. Negative interaction and satisfaction with social support among older adults. Journal of Gerontology: Psychological Science, v. 50B, p. 59-73, 1995.

LEE, G. R.; NETZER, J,K, Depressionamong older parents: the role of intergenerational exchange. Journal of Marriage and Family, n. 57, p. 823-833, 1995.

LIANG, J.; KRAUSE, N. M.; BENNETT. J. Social exchange and well-being: is giving better than receiving? Psychology and Aging, n. 16, p. 511-523, 2001.

MCCULLOCH, B. J. The relationship of intergenerational reciprocity of aid to the morale of older parents: equity and exchange theory comparisons. Journal of Gerontology: Social Sciences, n. 45B, p. 15-155, 1990.

NERI, A. Qualidade de vida e idade madura. São Paulo: Papyrus, 1993.

PILLEMER, K.; GLASGOW, N. Social integration and aging: background and trends. In: PILLEMER, K.; MOEN, P.; WETHINGTON, E.; GLASGOW, N. (Ed.). Social integration in the Second half of life. Baltimore: Johns Hopkins University Press, 2000.

RAMOS, L. R. Family support for elderly people in Sao Paulo, Brazil, In: KENDING, L.; HASHIMOTO, A. (Ed.). Family support for the elderly: the international experience. London: Oxford University Press, 1992. p. 225-232.

RAMOS, L. R.; SAAD, P. Morbidity among the aged. In: SEADE Fundacao Sistema Estadual de Analise de Dados (Ed.). Profile of the elderly in São Paulo. São Paulo: SEADE, 1990, p. 161-172. 
RIO GRANDE DO SUL. Conselho Estadual do Idoso. Os idosos no Rio Grande do Sul: estudo multidimensional de suas condições de vida - relatório de pesquisa. Porto Alegre: CEI, 1997.

ROOK, K. S. Reciprocity of social exchange and social satisfaction among older women. Journal of Personality and Social Psychology, EUA, n. 52, p. 145-154, 1987.

STOLLER, E. P. Exchange patterns in the informal networks of the elderly: the impact of reciprocity on morale. Journal of Marriage and the Family, EUA, n. 47, p. 335-342, 1985.

UNITED NATIONS. Program of United Nations for the Development. Zero Hora, 1998.

WILMOTH, J. M. Unbalanced social exchanges and living arrangements transitions among older adults. The Gerontologist, v. 40, n. 1, 64-74, Feb. 2000.

Recebido em: setembro de 2005

Aceito em: junho de 2006 